\title{
Statistical investigation on the power-law behavior of magnetic fluctuations in the Venusian magnetosheath
}

\author{
Navin Kumar Dwivedi ${ }^{*}$ (D), Daniel Schmid ${ }^{1,2}$, Yasuhito Narita ${ }^{1}$, Peter Kovács ${ }^{3}$, Zoltan Vörös $s^{1,4}$, Magda Delva ${ }^{1}$ \\ and Tielong Zhang ${ }^{1}$
}

\begin{abstract}
The present work aims to develop a better understanding of wave and turbulence processes in the planetary magnetosheath region. We study the plasma conditions (temperature, flow velocity, and magnetic field), the low-frequency wave properties, and the energy spectra for magnetic field fluctuations in the Venus magnetosheath. We use the magnetic field data of 101 magnetosheath flank crossings from the Venus Express magnetometer experiment in the years 2006 and 2008. The statistical investigation of the plasma conditions shows that the mean magnetic field amplitude is about $10 \mathrm{nT}$, the average proton temperature of the order of MK, and the super-Alfvénic, subsonic bulk plasma flow. Below $0.07 \mathrm{~Hz}$, the angle of propagation is about $80^{\circ}$ for the most of the cases, and it varies from $10^{\circ}$ to $90^{\circ}$ above the frequency $0.07 \mathrm{~Hz}$. The compressibility shows similar distribution at low (below $0.07 \mathrm{~Hz}$ ) and high frequencies (above $0.07 \mathrm{~Hz}$ ). The energy spectra in the spacecraft frequency frame reveal the power-law behaviors which give physical insight on the energy transfer from larger to smaller scales due to wave-wave interaction. A spectral break (sudden change in slope) is observed at $0.25 \mathrm{~Hz}$, above which the spectral curve becomes steeper with spectral indices between -4 and -1.5 (close to the Kolmogorov slope, $-5 / 3$ ). The low-frequency part (below $0.07 \mathrm{~Hz}$ ) having a spectral index close to -1 indicates the energy cascades due to mirror mode waves, and the steepen spectra at high frequencies (above $0.07 \mathrm{~Hz}$ ) with spectral indices between -4 and -0.5 are interpreted as the energy accumulation due to mirror mode and proton cyclotron waves.
\end{abstract}

Keywords: Turbulence; Waves; Magnetosheath; Power spectral density; Power law

\section{Background}

The plasma environment around Venus serves as a natural laboratory to study wave activity and turbulence caused by the solar wind interaction with the nonmagnetized planetary body. Venus does not have its own intrinsic magnetic field. The supersonic solar wind bears a magnetic field and electric field. This electric field plays a crucial role in the removal of the planetary atmosphere from an unmagnetized planet. The ultraviolet radiations coming from the sun ionizes the upper atmosphere of the planet creating an ionosphere. The ionosphere hinders the solar wind when the thermal pressure of the

\footnotetext{
*Correspondence: navin.dwivedi@oeaw.ac.at

1 Space Research Institute, Austrian Academy of Sciences, Schmiedlstrasse 6, Graz, Austria

Full list of author information is available at the end of the article
}

ionosphere exceeds the solar wind dynamic pressure. A magnetic barrier is being created in the form of magnetic cover on the ionosphere, which deflects the solar wind and form a detached bow shock. The ionosphere despite having strong thermal pressure deflects the solar wind, while some magnetic flux still manage to sink into the ionosphere and provides the environment to study in situ measurements and turbulence. When the solar wind dynamic pressure exceeds the thermal pressure of the ionosphere magnetic field, the plasma is convected downwards into the ionosphere and creates a global magnetic field. The magnetic field lines of the solar wind wrap around the ionospheric obstacle and construct an induced magnetosphere (Zhang et al. 2008). The induced magnetosphere is inherent of dayside magnetic obstacle to the solar wind which is defined by a surface

\section{黛 Springer}

(c) 2015 Dwivedi et al. Open Access This article is distributed under the terms of the Creative Commons Attribution 4.0 International License (http://creativecommons.org/licenses/by/4.0/), which permits unrestricted use, distribution, and reproduction in any medium, provided you give appropriate credit to the original author(s) and the source, provide a link to the Creative Commons license, and indicate if changes were made. 
called the ionopause, and the magnetotail. Even though Venus has some close resemblance with Earth such as the heliocentric distance approximately $0.72 \mathrm{AU}$ which is $1 \mathrm{AU}$ for Earth, and the planet radius approximately about $R_{\mathrm{V}}=6053 \mathrm{~km}$ which is $R_{\mathrm{E}}=6371 \mathrm{~km}$ for Earth, the induced magnetosphere of Venus has a different spatial scale size from the characteristic scales of the Earth magnetosphere.

The solar wind dynamic pressure and the thermal pressure of the ionospheric plasma balance each other at the ionopause surface. Bow shock formation occurs at upstream of the obstacle whose location changes with the solar cycle. The subsolar bow shock location is observed at about $1900 \mathrm{~km}$ from the surface of the planet; however, the terminator bow shock is detected at 12,953 km by Venus Express during the solar minimum. In the immediate post-shock flow, Venus has a magnetosheath region just like that of any other magnetized planet. In the early 1960s, Venus was the primary object to explore and more than 20 spacecraft missions were in operation. The most significant knowledge about the solar wind interaction with the Venusian environment was provided by the Pioneer Venus Orbiter (PVO) mission (Russell et al. 1980). A plasma mantle region forms between the magnetosheath and ionosphere, which is a mixture of the solar wind and ionospheric plasmas. One of the main results from the PVO mission is the detection of wave activity in the plasma mantle near the ionopause (Strangeway and Russel 1996). The hot magnetized plasma in the magnetosheath is separated from the thermal plasma of the ionosphere by the ionopause. Upstream waves are studied at Venus' foreshock (Russell et al. 1982) and at Venus' bow shock (Orlowski et al. 1994). Moreover, post-terminator waves are studied (Brace et al. 1982; Russell et al. 1982; Scarf et al. 1985) by using PVO data. The solar wind flow around Venus is discussed (Biernat et al. 2005) within the frame of magnetohydrodynamics, and linearly polarized transverse waves (Luhmann 1995) are studied in the quasi-parallel bow shock. Many aspects of the Venus plasma environment such as the bow shock location and the magnetosheath thickness are governed by the solar activity and vary with the phase of the solar cycle. The Venus Express spacecraft (Titov et al. 2006) was inserted into polar orbit in April 2006. The orbit of Venus Express makes it possible to study using in situ measurements the solar wind interaction with the ionosphere because of its low periapsis altitude. Venus Express also enhanced the measurement resolution and permits better exploration of the near-subsolar bow shock. Extensive exploration of the Venus day and night side magnetosheath now gives a new opportunity to study the nature of turbulent magnetic field fluctuations.

Proton cyclotron waves are observed for the first time at Venus (Delva et al. 2008a, b) which give the evidence of the escape of hydrogen from the exosphere of Venus by the pick up process. Delva et al. (2008a, b) further report that proton cyclotron waves are clearly observed up to about $5 \mathrm{R}_{\mathrm{V}}$ upstream of the bow shock. Volwerk et al. (2008a, b) perform case studies and statistical investigations to identify the compressive waves in the Venus magnetosheath using magnetometer data from Venus Express spacecraft (Zhang et al. 2006). Volwerk et al. (2008a, b) report that the compressive waves propagate perpendicular to the mean magnetic field and show similar properties to the mirror mode waves, which are often observed in various magnetospheric regions of the planetary objects, e.g., Earth's magnetotail (Rae et al. 2007), Earth's magnetosheath (Baumjohann et al. 1999; Constantinescu et al. 2003; Lucek et al. 1999; Tsurutani et al. 1982), the ion pick up region near Io (Huddleston et al. 1999), and the magnetosheath of Jupiter (Erdös and Balogh 1993) and Saturn (Bavassano Cattaneo et al. 1998). Volwerk et al. (2008a, b) also conclude that the mirror mode waves detected in the Venus magnetosheath have periods between 5 and $15 \mathrm{~s}$, and the probability to observe the mirror mode waves is higher at the dayside magnetosheath and is lower in the nightside magnetosheath. Vörös et al. (2008) statistically examine the properties of the magnetic field fluctuations in Venus' wake and magnetosheath regions. They exploit Venus Express spacecraft data for computing the energy spectra and implement the wavelet technique in order to identify the spectral scaling ranges and indices. Three distinct spectral indices are observed. The energy spectra in the dayside magnetosheath show $\frac{1}{f}$ spectral scaling indicating the existence of multiple sources of fluctuations. The magnetic field fluctuations in the nightside wake exhibit power-law energy spectra with the spectral index approximately -2.5 . This indicates the presence of turbulence in the wake region, which is strongly dissipative in nature. Vörös et al. (2008) also find the turbulence fluctuations and wavy structures near the terminator. Guicking et al. (2010) study the wave properties of low-frequency magnetic field fluctuations in the Venus magnetosheath and mid-magnetotail regions. They apply an analytical streamline model to examine the plasma flow around the Venus and the spatial distributions of wave intensity of the magnetic fluctuations. Guicking et al. (2010) find an enhancement of the wave intensity in the dayside magnetosheath and a decrease towards the terminator. Guicking et al. (2010) further perform a comparative study between Venus and Mars and conclude that the spatial distribution of wave properties detected in the Venus dayside and nightside magnetosheath, the tail and the mantle regions show similar characteristic behavior as observed at Mars. The observations also support the idea of convection of waves by plasma flow. 
The present work emphasizes the statistical study of the plasma conditions, the characteristics of the low frequency waves, the energy spectra, and associated spectral indices in spirit of revealing the turbulence mechanism in the Venus magnetosheath. We use the data obtained by Venus Express magnetometer (VEXMAG) (Zhang et al. 2006) and plasma instrument Analyser of Space Plasma and Energetic Atoms (ASPERA) (Barabash et al. 2007) in the present investigation. The magnetosheath is composed of plasma, which is highly disturbed with large-amplitude variations in the velocity, magnetic field, temperature, and density. These disturbances in the plasma can contribute to particle acceleration and turbulence. The magnetosheath is a natural laboratory to study compressive and non-compressive turbulence. Temporal variations of the magnetic field in the magnetosheath may represent many wave modes. In the present work, we investigate the spectral features of the magnetic field fluctuations in magnetosheath and we find that the magnetic field fluctuations are about to evolve into turbulence. We also discuss the properties of low-frequency waves, e.g., compressibility and the angle of propagation. The present study is complementary to the previous investigations (Guicking et al. 2010) in the sense that the previous work is mainly focused on the wave properties, whereas the present investigation focuses on the scaling properties in the magnetosheath energy spectra.

The solar wind interaction with the ionosphere and magnetosphere of the planetary bodies is unique in many respect but also exhibits a continuum of possible interactions. During the solar wind interaction with the highly electrically conducting ionosphere, the magnetic field deflects the supersonic solar wind. In spite of dominance of the magnetic field in deflecting the solar wind flow, the ionosphere which is actively coupled with the outer magnetosphere by field aligned current plays a significant role in several magnetospheric processes. In case of Jupiter despite having strong magnetic field, the centrifugal force governs many aspects of the Jovian magnetosphere-solar wind interaction. Mercury has strong magnetic field and no ionosphere in contrast to the Earth. Venus has no intrinsic magnetic field and therefore solar wind directly interacts with the ionosphere. The dissimilarities between the terrestrial and Venus' ionosphere and magnetosphere are crucial to understand the fundamental physical processes occurring within each of them. In many manners, the physical processes at Earth and Venus represent specific cases in which the fundamental concepts and models can be put on the examination. The induced magnetopause of Venus has altitude of $300 \mathrm{~km}$ at subsolar point and $1013 \mathrm{~km}$ at the terminator; however, the width of the Earth magnetosheath is about $30,000 \mathrm{~km}$ at subsolar point. Regardless of differences in the magnetosheath size, the waves and fluctuations appear equitably in the Venus and Earth magnetosheath (Luhmann et al. 1983, 1986). Venus, in spite of having limited magnetosheath width, does have presumably small Reynolds number featuring the turbulent flow in the magnetosheath. The understanding of the wave activities and turbulence processes in the Venus magnetosheath will also enable us to predict the possible conditions in the terrestrial magnetosphere when the magnetic field is weak. The study at Venus is also applicable to comets. The statistical investigation and analysis related to wave and turbulence in the Venus magnetosheath will allow us to make educated foresights to the observational test at Mars and comets, which would be helpful to deepen our understanding related to waves and turbulence globally.

\section{Methods}

We perform a statistical analysis of magnetic energy spectra in the frequency domain. The primary data set includes $1 \mathrm{~Hz}$ magnetic field data recorded by VEXMAG instrument on board Venus Express. The $1 \mathrm{~Hz}$ data are obtained by averaging over high-resolution sampling at $32 \mathrm{~Hz}$ to suppress the disturbance magnetic field of the instrumental and spacecraft origins. The energy spectra are evaluated from the magnetic field data. The secondary data set is the ion moment data (density, flow velocity, and temperature) obtained by the Analyser of Space Plasma and Energetic Atoms (ASPERA) instrument at a time resolution of 3-min sampling. The ion data are used to assist the scientific interpretation of the energy spectra including (1) conversion of the temporal scales into the spatial scales using Taylor's frozen-in hypothesis and (2) estimation of the Alfvén speed $\left(V_{A}\right)$, sound speed $\left(c_{S}\right)$, and the gyrofrequency $\left(\Omega_{i}\right)$ and gyroradius $\left(\rho_{i}\right)$ for thermal ions.

The data are used to investigate the turbulence properties in the Venus magnetosheath which are not addressed in detail in previous study (Guicking et al. 2010). One hundred one (101) data sets are selected out of 5 months from January to May in each year from 2006-2008. From every data set, a 10-min interval in the magnetosheath is selected. Ten minute is a typical time length for quasistationary fluctuations avoiding multiple boundary crossings. Instead of using an automatic procedure for the selection of magnetosheath events, we closely investigate each of the magnetic field time series data to select the 10min magnetosheath intervals out of 101 magnetosheath crossings. In order to select the 10-min intervals in the magnetosheath, we first analyze the magnetic field time series data and we observe an abrupt change in the magnitude of the magnetic field as an indication of bow shock crossing. Just after the bow shock crossing, we find highamplitude magnetic field fluctuations as a signature of magnetosheath crossing, and select the 10-min interval of quasi-stationary fluctuations in the magnetosheath. A 
typical example of a selection of 10-min data in the magnetosheath is illustrated in Fig. 1. The selected 10-min magnetosheath interval of the interest is shown (Fig. 1a) by the horizontal black bar $(02: 40: 06$ to $02: 48: 38 \mathrm{UT})$. The zoom out of the selected event of $10 \mathrm{~min}$ is illustrated in the bottom panel (Fig. 1b).

The magnetic field data are first represented in the Venus Solar Orbital (VSO) coordinate in which the $x$ axis is in the direction to the sun, the $y$-axis points in the opposite direction of reference frame of the planetary orbital motion, and the $z$-axis is pointing northward from the orbital plane (in the same sense as that of geocentric solar ecliptic coordinate system). The magnetic field data are then transformed into the mean-field-aligned (MFA) coordinate system with respect to the large-scale magnetic field direction in which the $z$-axis is defined as the direction $\left(\mathbf{e}_{\mathbf{z}}\right)$ of the mean magnetic field, the cross product $\left(\mathbf{e}_{\mathbf{y}}\right)$ of $\hat{\mathbf{X}}_{\mathrm{VSO}}$ with the new $z$-axis gives the $y$-axis, and the $x$-axis completes the right-handed coordinate system $\left(\mathbf{e}_{\mathbf{x}}\right)$ :

$$
\begin{gathered}
\mathbf{e}_{\mathbf{x}}=\mathbf{e}_{\mathbf{y}} \times \mathbf{e}_{\mathbf{z}} \\
\mathbf{e}_{\mathbf{y}}=\hat{\mathbf{X}}_{\mathrm{VSO}} \times \frac{\mathbf{B}_{\mathbf{0}}}{B_{0}}
\end{gathered}
$$

$$
\mathbf{e}_{\mathbf{z}}=\frac{\mathbf{B}_{\mathbf{0}}}{B_{0}}
$$

where $B_{0}$ is the mean magnetic field.

The power spectral densities (PSDs) are evaluated using the Welch algorithm (Welch 1967) in the frequency range 0.008 to $0.5 \mathrm{~Hz}$ for 101 time intervals

$$
P_{i j}\left(f_{s c}\right)=\frac{1}{T}\left\langle\tilde{B}_{i}\left(f_{s c}\right) \tilde{B}_{j}^{\dagger}\left(f_{s c}\right)\right\rangle
$$

where $i$ and $j$ are the magnetic field components, $T$ denotes the time length, $\tilde{B}$ the Fourier transform of the magnetic field vector (as a function of the frequencies), and the bracket \langle\rangle is the operation of averaging over time subintervals of about 2-min sliding windows with $50 \%$ overlap. The power spectral density is a $3 \times 3$ matrix in which the diagonal elements $P_{11}$ and $P_{22}$ are the perpendicular components $P_{\perp x}$ and $P_{\perp y}$ of the power spectra and $P_{33}$ is the parallel component $P_{z}$ of the power spectra. Figure 2a represents an typical example of the computed magnetic field spectra from the magnetosheath interval shown in Fig. 1.

To determine the wave properties like ellipticity, polarization, wave power, and propagation direction, the spectral analysis method (Arthur et al. 1976) with three

21May, 2008

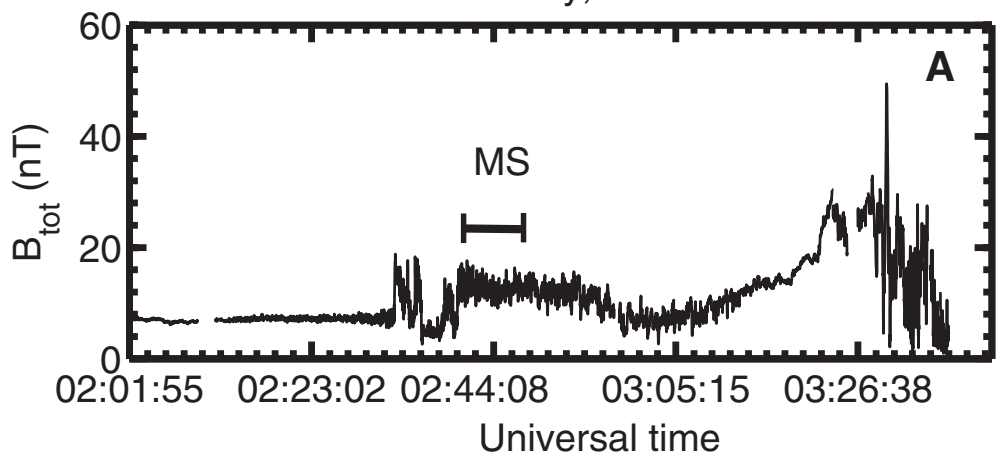

21 May, 2008

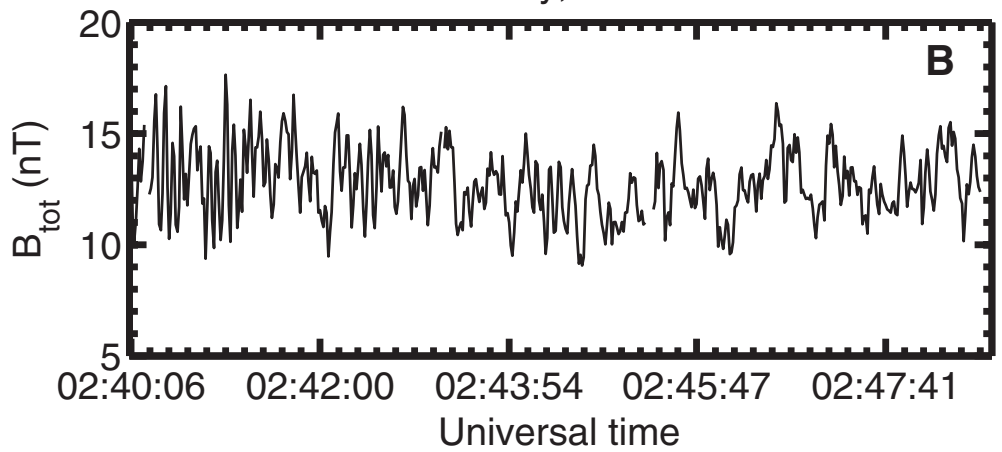

Fig. 1 Time series plot of the magnetic filed magnitude for the Venus magnetosheath crossing of Venus Express on 21 May, 2008 (a), the zoom out (b) of the selected event of 10 minute in the magnetosheath 

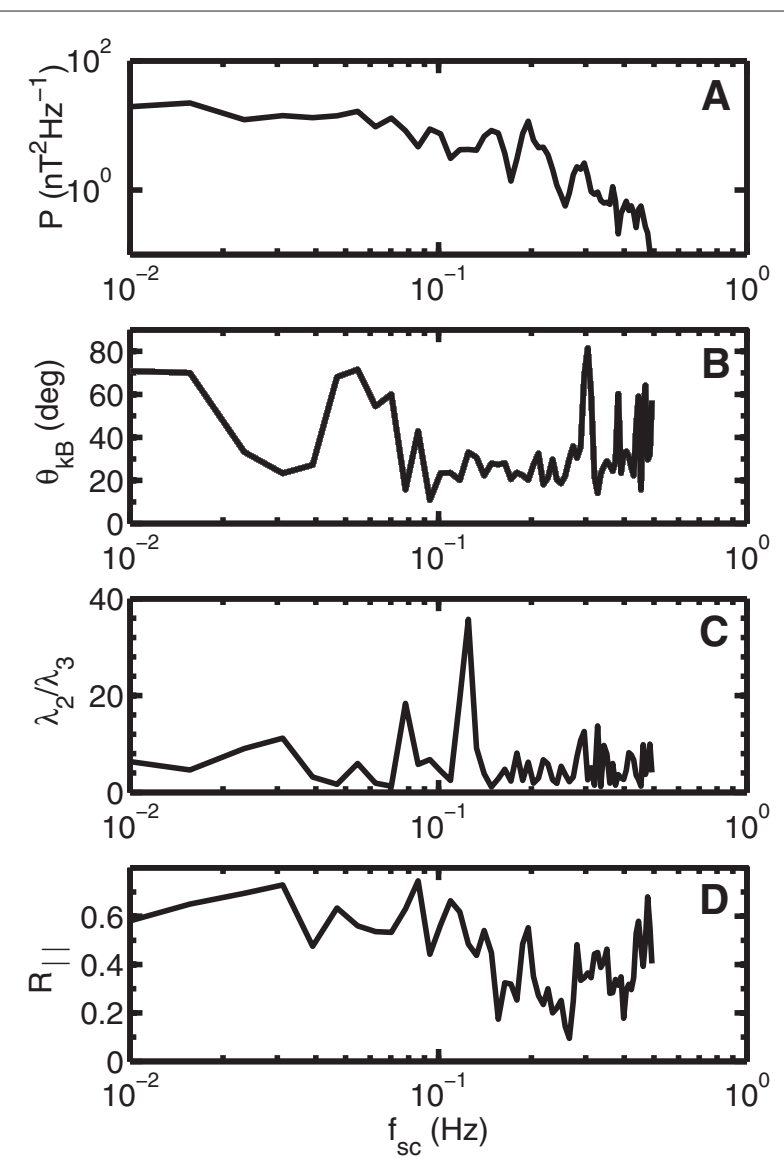

Fig. 2 Typical example. Magnetic energy spectrum for the magnetic field fluctuations in the Venus magnetosheath on 21 May, 2008 (cf. Fig. 1) (a), the angle between the wave vector and the mean magnetic field in degrees (b), the ratio of the intermediate to the smallest eigenvalues obtained by minimum variance analysis (c), and the magnetic compressibility (d) as a function of the frequencies in the spacecraft frame

different techniques is used. The power spectral density matrix is a complex number and therefore has real and imaginary parts. In the present investigation, we use the technique developed by McPherron et al. (1972) in which the diagonalization of the real part of the power spectral density matrix gives the three eigenvectors $\left(\zeta_{1}, \zeta_{2}, \zeta_{3}\right)$ and corresponding eigenvalues $\left(\lambda_{1}, \lambda_{2}, \lambda_{3}\right)$ for maximum, intermediate, and minimum variance directions, respectively. The minimum variance direction provides the propagation direction of the wave. We point out that the propagation direction of the wave is only well determined if the intermediate eigenvalue is sufficiently large compared with the minimum eigenvalue. To determine the wave vector direction, we here apply the condition $\lambda_{2} / \lambda_{3} \geq 5$ at each frequency of the 101 events. We find 64 events which satisfy the condition $\lambda_{2} / \lambda_{3} \geq 5$ at least at one frequency and then calculate the angle $\left(\theta_{k B}\right)$ between the wave propagation and the mean field at each frequency. We define the compressibility as the ratio of the compressive power $\left(P_{\|}\right)$to the total power $P_{\text {total }}=P_{\|}+P_{\perp}$ (where $P_{\perp}$ is the transverse power), which are determined from the power spectral density matrix. Figure $2 \mathrm{~b}-\mathrm{d}$ respectively shows the change in the calculated angle $\left(\theta_{k B}\right)$ between the wave propagation and the mean field, the variability in the eigenvalue ratio $\left(\lambda_{2}\right.$ and $\lambda_{3}$ ) under a condition $\lambda_{2} / \lambda_{3} \geq 5$, and the variation in the compressibility with respect to frequency for the example case in Fig. 1.

In the terrestrial case, various regions of the magnetosheath exhibit different properties of the waves and turbulence, depending on the position in relation to the bow shock and magnetopause. The Venus Express remains mostly in the solar wind. The inbound and outbound bow shock crossings hardly appear in an interval of 1 to $2 \mathrm{~h}$ around the periapsis. In order to determine the spacecraft position in the Venus magnetosheath, we use the model developed by Zhang et al. (2008) and exploit the equation for the conic section to plot the bow shock and magnetopause positions:

$$
R_{M S}=\frac{2.169505}{1+0.61733 \cos \theta}\left(0^{\circ} \leq \theta \leq 120^{\circ}\right)
$$

where $\theta$ is the solar zenith angle. We identify and plot the spacecraft positions in the magnetosheath for the selected events. Figure 3 displays the locations of the selected events of interest in the magnetosheath.

In order to investigate the spectral behavior of the magnetosheath turbulence, we construct the following automatic numerical procedure to determine the suitable frequency ranges and the power law indices for the magnetic energy spectra. We compute the mean of power spectral density (PSD) in sliding frequency bins of an equal logarithmic size $(\Delta(\log (f))=0.05)$ as a function of the mean frequencies. Thus a new series of $\log (f)$ and $\log (P)$ datum pairs are generated where the independent variables equidistantly follow each other on the logarithmic scale. Then, linear relation is fitted to fivepoint sliding segments with $30 \%$ overlapping of the new series and the obtained slope values are recorded in terms of the mid frequencies of the segments. Finally, the spectral ranges are selected between frequencies for which the variation of the slopes remain within a certain limit, $L$. In our case, the limit is given as $L=0.4 R$, where $R$ stands for the whole range of the obtained slope values.

\section{Results and discussion}

Figure 4 shows the basic plasma parameters and their variations in the present statistical investigation. Figure 4a displays the histogram of the background magnetic field. In most of the cases, the background magnetic field has a 


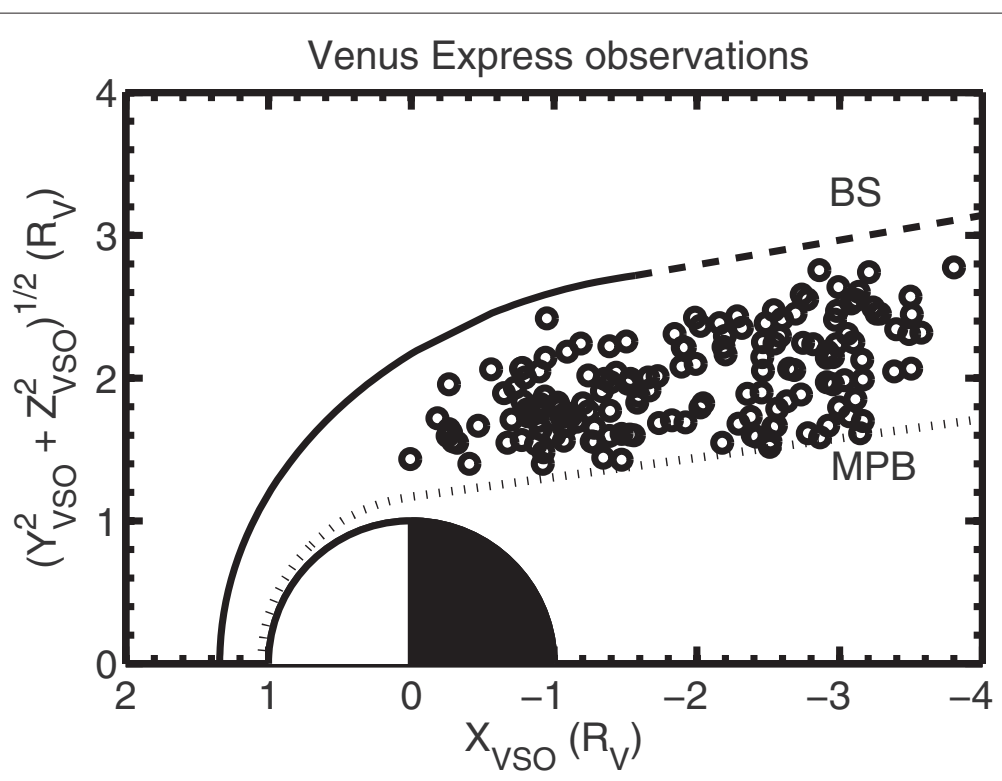

Fig. 3 Event location in the magnetosheath. Spatial coordinates of 101 magnetosheath crossing events of Venus Express in 2006-2008 are represented in the coordinate system spanning the Venus-to-Sun direction on the $x$-axis (in the Venus-Solar-Orbital system) and the vertical distance from the $x$-axis assuming the axi-symmetry of the magnetosheath shape around the $x$-axis. The bow shock position is determined by Eq. 5 up to $-1.5 R_{v}$. The bow shock location (with an abbreviation BS) is presented by the black solid line up to $-1.5 R_{v}$ and is further extended up to $-4 R_{v}$ and illustrated by dash line, and the magnetic pile up boundary is illustrated with an abbreviation MPB

value of about $10 \mathrm{nT}$. Figure $4 \mathrm{~b}, \mathrm{c}$ shows the variation of the ion temperature and ion flow velocity, respectively.

Figure 5a-d manifests the histogram of the ion cyclotron frequency, the thermal ion gyroradius, Alfvén Mach number (ratio between ion flow velocity $V_{\text {flow }}$ and Alfvén speed $V_{A}$ ), and sonic Mach number (ratio between ion flow $V_{\text {flow }}$ velocity and sound speed $c_{S}$ ) using the basic parameter values of the selected events, respectively. We find that the flow velocity in the magnetosheath is characterized as super-Alfvénic and subsonic. The sound speed and Alfvén speed is defined as:

$$
\begin{gathered}
c_{s}=\sqrt{\frac{\gamma p_{t h}}{\rho}} \\
V_{A}=\frac{B_{0}}{\sqrt{\mu_{0} n_{p} m_{p}}}
\end{gathered}
$$

where $c_{s}$ denotes the sound speed, $\gamma=\frac{5}{3}$ the polytropic index for $N=3$ (degree of freedom), $p_{t h}$ the thermal pressure, $\rho$ the mass density, $V_{A}$ Alfvén speed, $B_{0}$ the mean magnetic field, $\mu_{0}$ the permeability of free space, $n_{p}$ the proton density, and $m_{p}$ the mass of the proton.

The fluctuation energy is evaluated as PSD and fitted with a scale-dependent power-law. To compute the PSD, the magnetic field components on each time interval are transformed into the MFA coordinate system. This transformation gives the transverse and compressive power of the fluctuations with respect to the mean magnetic field. The data in the MFA coordinate system are Fourier transformed into the frequency space with the help of Welch algorithm and we compute the cross spectral density matrix. The total magnetic field $\left(B_{t}\right)$ power spectra is obtained by the trace of the cross spectral density matrix. Figure 6a illustrates the superposed power spectral density for the 101 analyzed intervals in the frequency range from 0.008 to $0.5 \mathrm{~Hz}$ in the spacecraft frame, the total power spectra in black solid line. Figure $6 \mathrm{~b}$ represents the median of the power spectrum, which is shown by black thick line and the upper and lower quartiles of the 101 events (dotted lines). A slight hump in the energy spectra is obvious in the median of the total power spectra as an indication of the spectral break. It is clear from Fig. 6b that there is a slight hump in the spectra between 0.25 and $0.4 \mathrm{~Hz}$ which leads to the steeper spectral slope in the latter part (high-frequency) of the PSD.

In order to study in detail about the difference in the power spectra between the low and high-frequency domains, we determine the scale-dependent spectral indices (power-law indices) of the evaluated total power spectra with the help of the automatic numerical procedure as presented in section "Methods". Figure 7 shows the histogram of the estimated spectral indices $(\alpha)$ at low frequencies $(0.008$ to $0.07 \mathrm{~Hz})$ and almost $90 \%$ of the total spectral index variations show the most dominating peak of spectral indices between -3 and -0.5 . At low frequencies, the most remarkable peak evidently appears at spectral index close to -1 , the secondary population also appears with smaller spectral 


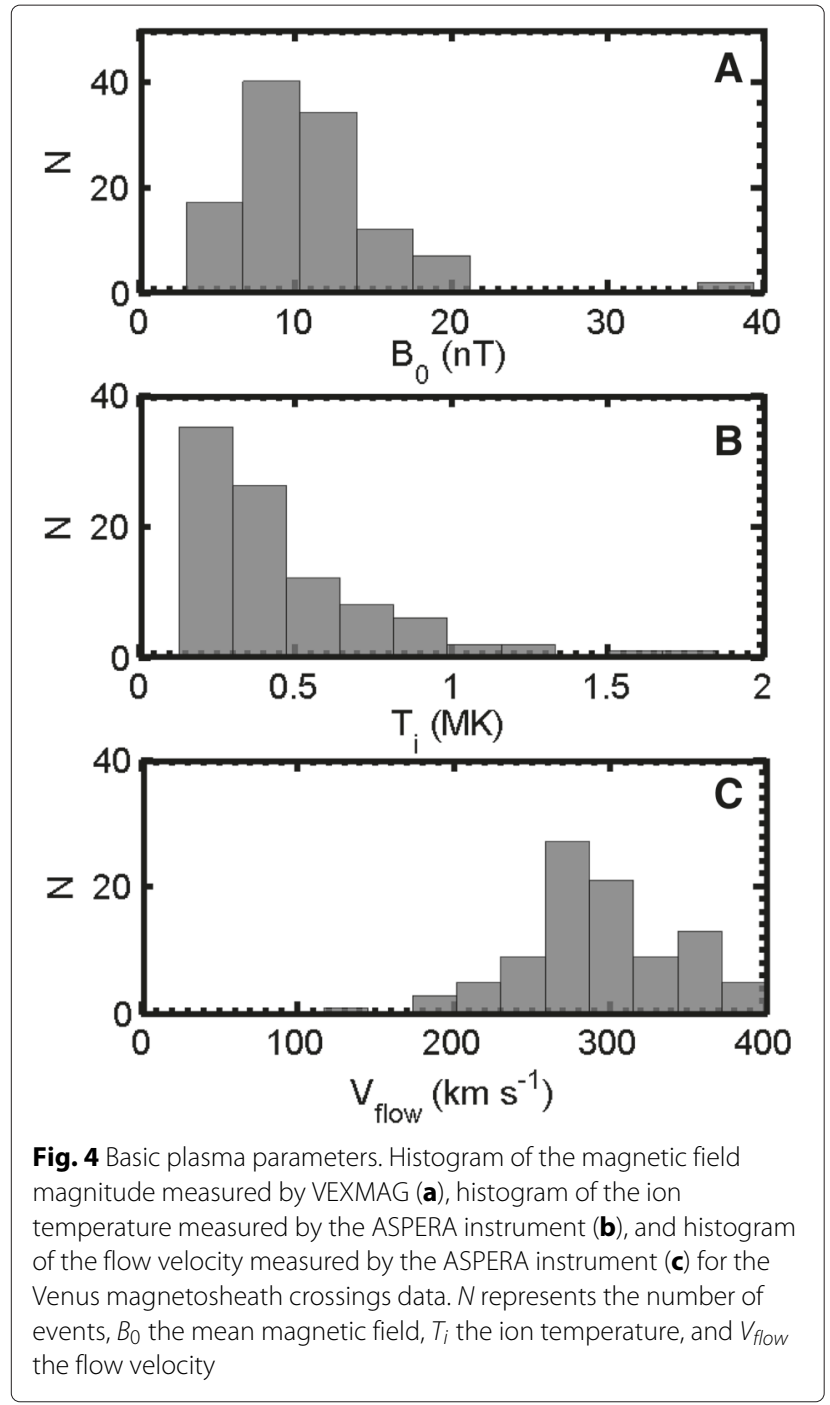

indices. Figure 8 illustrates the histogram of the spectral indices $(\alpha)$ at high frequencies (above $0.07 \mathrm{~Hz}$ ), and the frequency-dependent spectral indices vary between -4 and -0.5 for about $90 \%$ of the total slope occurrence. At high frequencies, once again the first prevailing peak of spectral index appears at about -1 , in addition to this the second dominating peak of spectral index exist at -1.5 and the third peak appears at around -2.2 .

The color-coded representation of two-dimensional histogram of the spectral indices in the Venus magnetosheath is shown in Fig. 9. The figure has three panels, the top panel includes frequency on the $\mathrm{x}$-axis, slope variation $(\alpha)$ is on the $y$-axis, and colors show the cumulative number of slope occurrences in logarithmically equal frequency ranges. The middle panel (Fig. 9) shows the number of occurrence of spectral slope values as a function of frequency, and the bottom panel (Fig. 9) represents the cumulative numbers of occurrence of power-laws as a

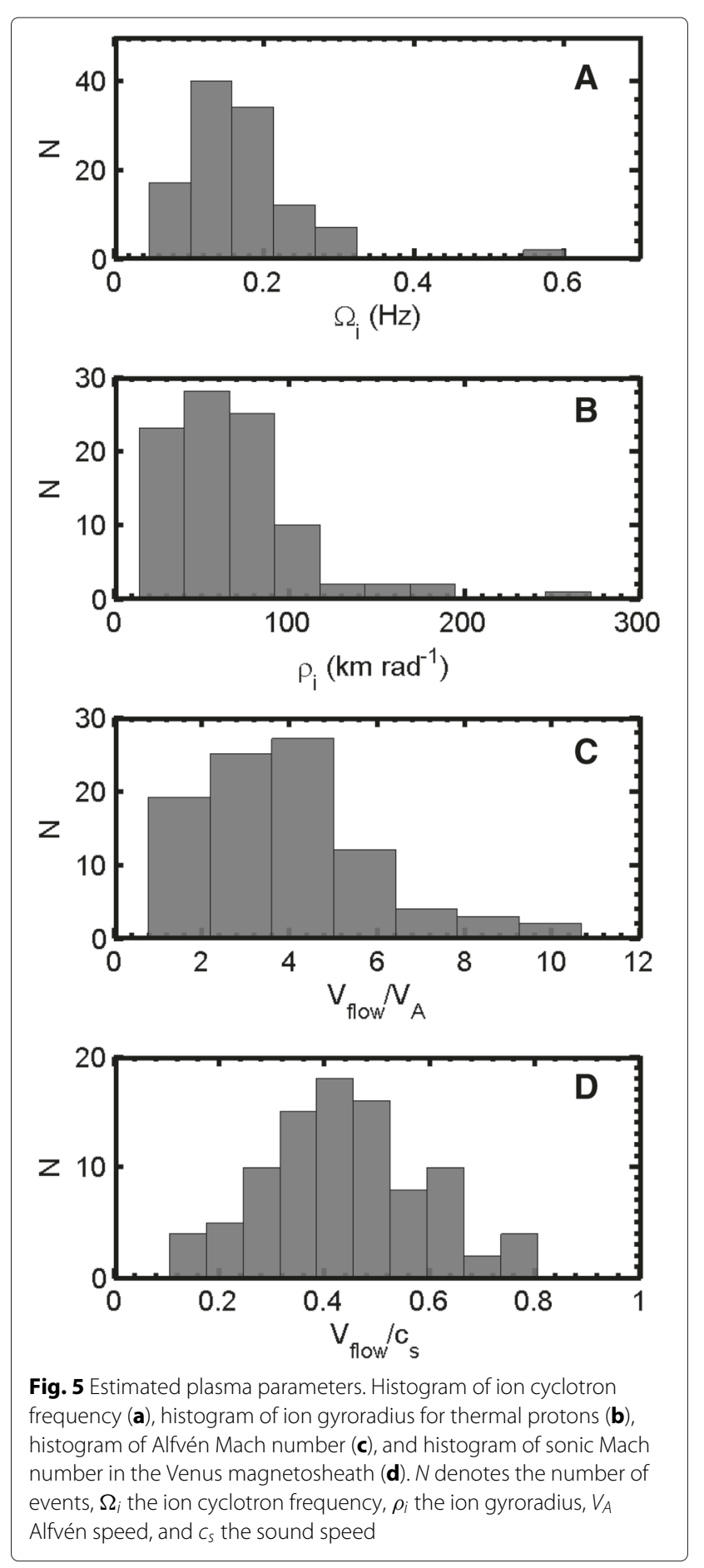

function of power-law indices $(\alpha)$. We further classify the top panel into four blocks (A, B, C, D) to quantitatively study the changes in the spectral indices in the twodimensional histogram in Fig. 9. The blocks A and B are limited to a frequency of $0.07 \mathrm{~Hz}$ (as indicated by an arrow on $x$-axis), and the blocks $C$ and $D$ start from that frequency. The blocks $\mathrm{A}$ and $\mathrm{C}$ are limited down to a power-law index of -2 (as indicated by an arrow on 


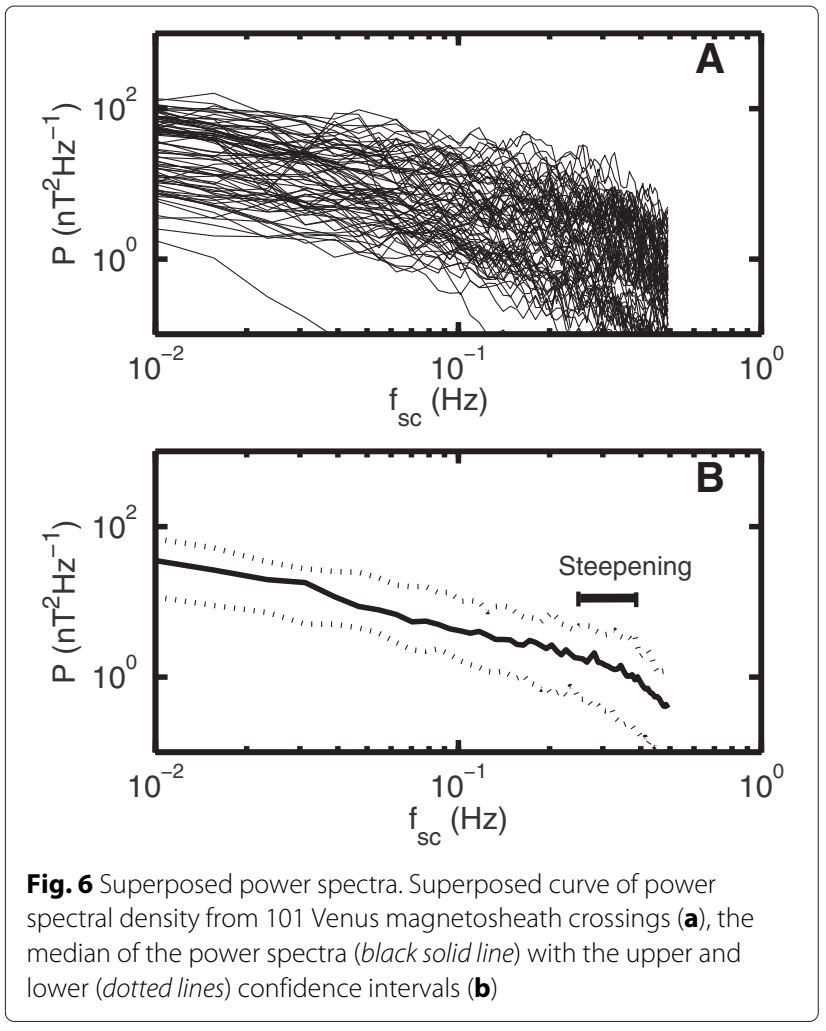

$y$-axis), and the blocks $\mathrm{B}$ and $\mathrm{D}$ have the indices smaller (steeper) than that index. We obtain the following results:

- In the block $\mathrm{A}$, the magnetic energy spectra have spectral indices between -2 and -0.5 for most of the cases.

- In the block B, there are few cases with the spectral indices smaller than -2 in the frequency range 0.008 to $0.07 \mathrm{~Hz}$.

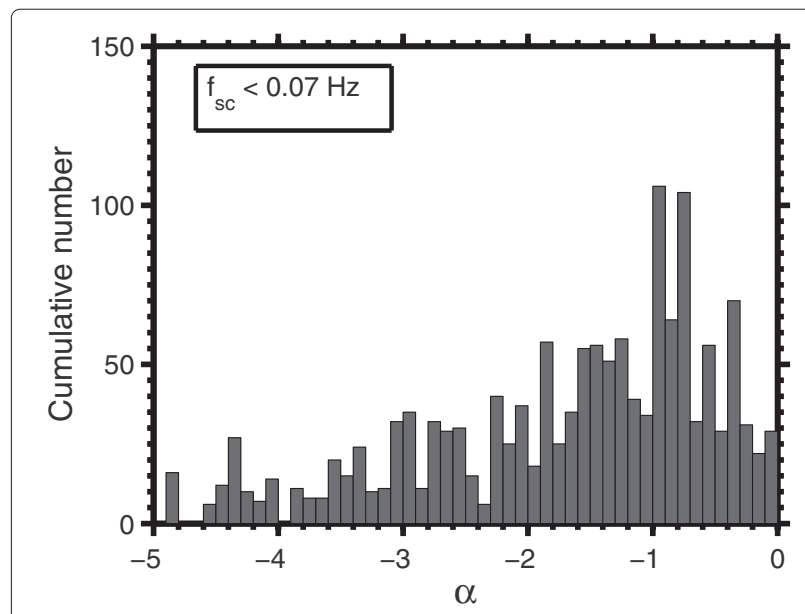

Fig. 7 Spectral slopes at low frequencies. Histogram of the spectral indices $(\alpha)$ below $0.07 \mathrm{~Hz}$

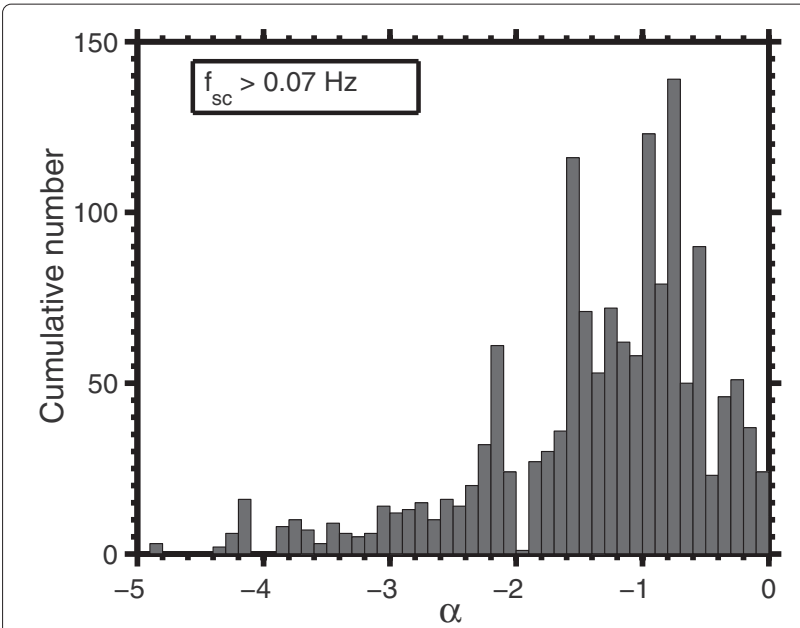

Fig. 8 Spectral slopes at high frequencies. Histogram of the spectral indices $(\alpha)$ above $0.07 \mathrm{~Hz}$

- In the block $C$, the spectral index varies from -2 to -0.5 . The majority of the population is at the index around -1 , and the second population with a spectral index close to -1.5 appears.

- In the block $\mathrm{D}$, there is a prominent peak of the histogram at an index of about -2.2 at frequencies $0.15 \mathrm{~Hz}$ and higher, and the signature of smaller spectral indices also appears.

- There is no slope detected beyond $0.4 \mathrm{~Hz}$ and therefore $0.4 \mathrm{~Hz}$ is the upper frequency limit of Fig. 9.

To summarize, the observed power-law is close to -1 at low frequencies (up to $0.07 \mathrm{~Hz}$ ). Above $0.07 \mathrm{~Hz}$, two additional steep power-laws appear, the first power-law exhibits a spectral index of -1.5 and the second powerlaw has the spectral index of -2.2 for the majority of the cases. However, as the analyzed frequencies are limited to $0.5 \mathrm{~Hz}$, the existence of the secondary power-law indices at higher frequencies cannot clearly be concluded. The results indicate that the spectral indices vary between -4 and -0.5 . A similar kind of spectral behavior and spectral indices are reported in the past study (Vörös et al. 2008) for several Venus magnetosheath events.

In addition, we study the presence of compressive or non-compressive waves. To determine the wave power and propagation direction, the spectral analysis method (Arthur et al. 1976) is widely used. Figure 10a displays the variation in the angle $\left(\theta_{k B}\right)$ between wave propagation direction and the mean magnetic field at low frequencies $(0.008$ to $0.07 \mathrm{~Hz})$. The propagation angle $\left(\theta_{k B}\right)$ distribution at low frequencies is mainly dominated by angle greater than $60^{\circ}$. We find more cases indicating the wave vector quasi-perpendicular to the mean magnetic field with the propagation angle $\left(\theta_{k B}\right)$ of about $90^{\circ}$, and there is no event with $\theta_{k B}$ smaller than $60^{\circ}$. It is also clear in Fig. 10b that in $60 \%$ of the cases, the propagation 

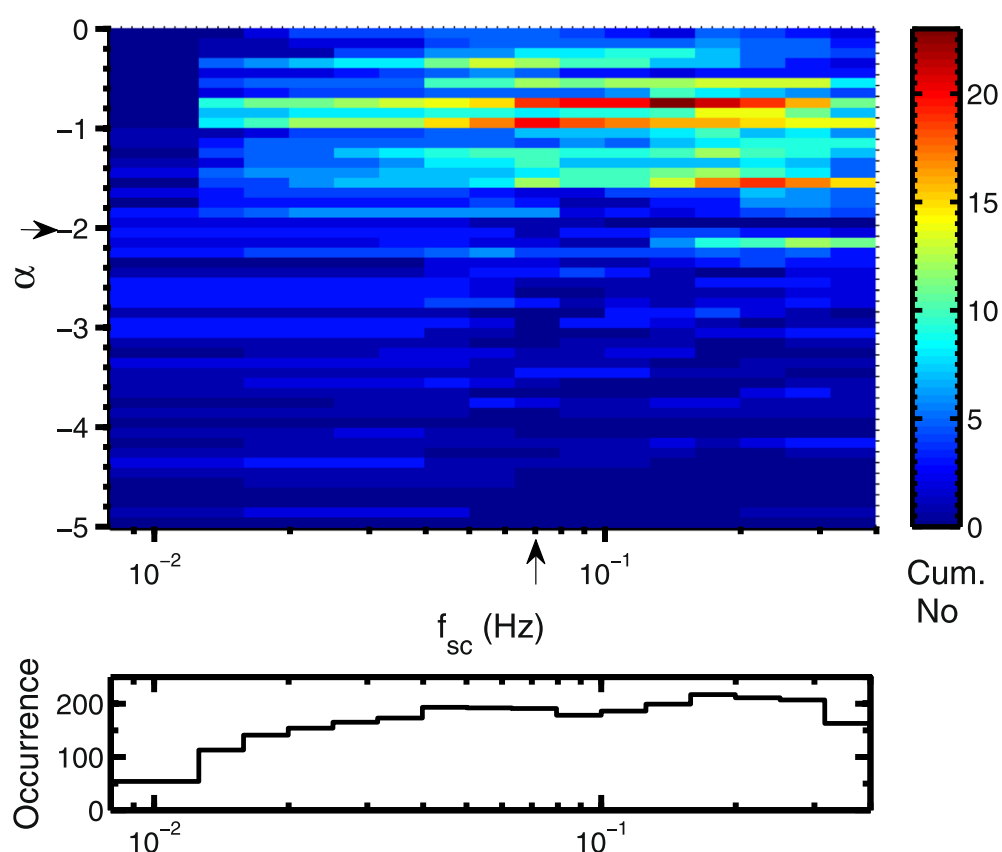

No

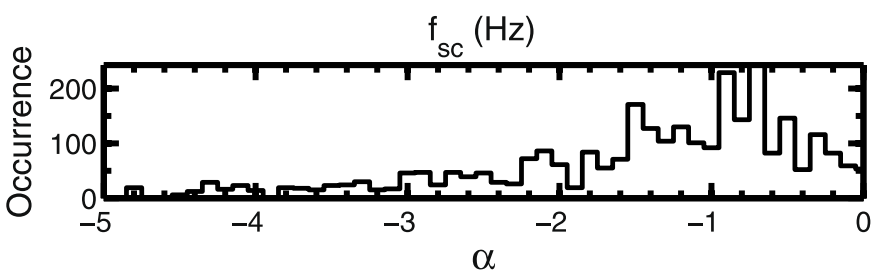

Fig. 9 2-D Histogram of spectral slope values. Two-dimensional histogram of the power-law indices $(\alpha)$ of the magnetic energy spectra in the Venus magnetosheath (top panel), the reduced histograms as a function of the frequencies (middle panel) and as a function of the spectral indices (bottom panel)

angle is almost $90^{\circ}$. Figure 11a illustrates the histogram of the propagation angle $\left(\theta_{k B}\right)$ at high frequencies $(0.07$ to $0.5 \mathrm{~Hz}$ ), and the value of $\theta_{k B}$ varies from $10^{\circ}$ to $90^{\circ}$. Figure 11a shows two distinct distributions of $\theta_{k B}$ with one population of angle at about $80^{\circ}$, and the second appears at about $30^{\circ}$. It is also obvious from Fig. $11 \mathrm{~b}$ that for $60 \%$ of the cases, the propagation angle has the variation between $60^{\circ}$ and $90^{\circ}$, however in $40 \%$ of the cases, the propagation angle is smaller than $60^{\circ}$. Therefore, one can say that the histogram at low frequencies shows the occurrence of large angle $\left(\theta_{k B} \gg 60^{\circ}\right)$; however, the histogram at high frequencies clearly shows a wider distribution of propagation angle with the occurrence smaller angles $\left(\theta_{k B} \ll 60^{\circ}\right)$. We study the compressibility, $R_{\|}=\frac{P_{\|}}{P_{\text {total }}}$, the ratio of the compressive power $\left(P_{\|}\right)$to the total power $\left(P_{\text {total }}=P_{\|}+P_{\perp}\right)$ where $P_{\perp}$ is the transverse power which are determined from the cross spectral density matrix. Figure 12a, b displays the histogram of compressibility at low frequencies, and it is evident that in more than $50 \%$ of the cases the compressive component with more power is commanding over the non-compressive component. Figure 13a, b presents the histogram of compressibility at high frequencies and about $60 \%$ of the occurrence reveal the predominating nature of the non-compressive component. The compressibility $\left(R_{\|}\right)$is accordingly higher at low frequencies and smaller at high frequencies.

\section{Conclusions}

The findings of the present investigation are followings:

- The resulting energy spectra of total magnetic field show distinct spectral indices at low and high frequencies.

- At low frequencies (below $0.07 \mathrm{~Hz}$ ), the energy spectra have spectral index variations from -3 to -0.5 . The prominent peak appears at a spectral index close to -1 for the majority of the cases, and the secondary peaks also exist with smaller spectral indices.

- Above $0.07 \mathrm{~Hz}$, the resulting energy spectra show a power-law scaling with spectral indices between -4 


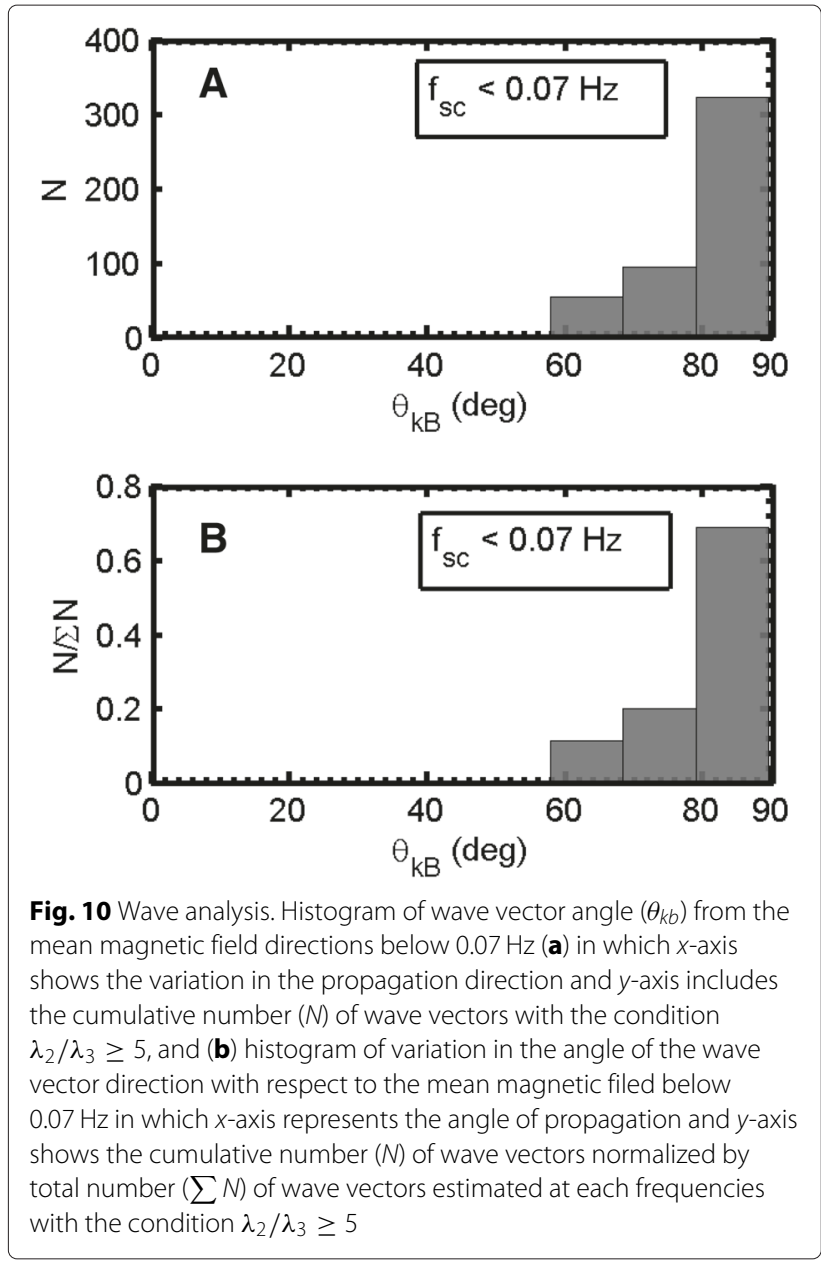

and -0.5 . In addition to obvious peak at spectral index -1 , two additional population of smaller spectral indices emerge at -1.5 and -2.2 .

- At about $0.3 \mathrm{~Hz}$, a steepening in the spectra is observed.

- The propagation angle and compressibility show different distributions at low and high frequencies.

- The propagation angle $\left(\theta_{k B}\right)$ is about $90^{\circ}$ for the majority of the cases, and more oblique to the mean magnetic field at low frequencies.

- The propagation angle $\left(\theta_{k B}\right)$ has wider distribution from $10^{\circ}$ to $90^{\circ}$ at high frequencies.

- There is more power in the compressive component at low frequencies, and less power in the compressive component at high frequencies.

In majority of the cases at low frequencies (below $0.07 \mathrm{~Hz}$ ), the energy spectra have spectral index close to -1 . At low frequencies, the compressibility is higher and the propagation direction is almost perpendicular to the mean magnetic field. According to Volwerk et al. (2008a, b), mirror mode structures have a period of $4 \mathrm{~s} \leq$
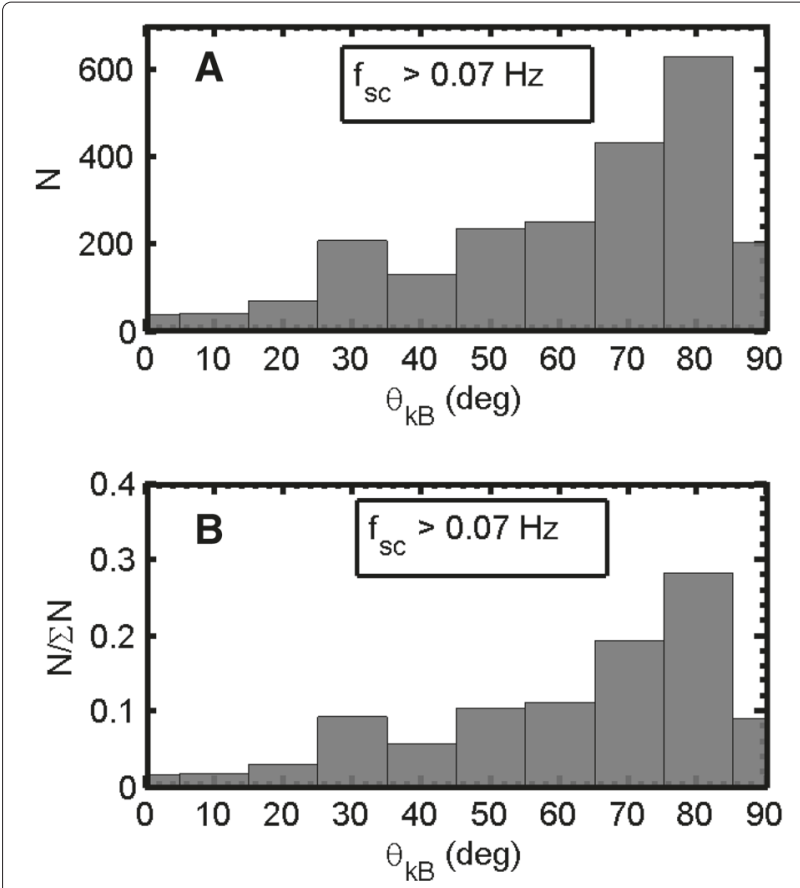

Fig. 11 Wave analysis. Histogram of wave vector angle $\left(\theta_{k b}\right)$ from the mean magnetic field directions above $0.07 \mathrm{~Hz}(\mathbf{a})$ in which $x$-axis shows the variation in the propagation direction and $y$-axis includes the cumulative number $(N)$ of wave vectors with the condition $\lambda_{2} / \lambda_{3} \geq 5$, and (b) histogram of variation in the angle of the wave vector direction with respect to the mean magnetic filed above $0.07 \mathrm{~Hz}$ in which $x$-axis represents the angle of propagation and $y$-axis shows the cumulative number $(N)$ of wave vectors normalized by total number $\left(\sum N\right)$ of wave vectors estimated at each frequencies with the condition $\lambda_{2} / \lambda_{3} \geq 5$

$T \leq 15 \mathrm{~s}$; therefore, the high value of compressibility and oblique propagation angle could possibly be a realization of accumulation of energy in the form of mirror mode structures at low frequencies. However, after $0.07 \mathrm{~Hz}$, the resulting energy spectra show the power-law scalings with spectral indices between -4 and -0.5 for the majority of the cases. The high-frequency part (above $0.07 \mathrm{~Hz}$ ) of the spectra is interpreted as turbulence and reveals the same behavior of power-law with break point frequency near $0.3 \mathrm{~Hz}$ in the spacecraft frame as observed in the solar wind (Bourouaine et al. 2012). A steepening in the spectra is observed with a spectral index variation from -4 to -1.5 . However, this steepening in the spectra is only over a very short range of frequencies $(0.3$ to $0.4 \mathrm{~Hz})$, but still it indicates similar characteristic behavior as observed in the magnetohydrodynamic (MHD) scale of the solar wind. At high frequencies (above $0.07 \mathrm{~Hz}$ ), the variation in the propagation angle $\left(\theta_{k B}\right.$ varies from $10^{\circ}$ to $\left.90^{\circ}\right)$, and compressibility could possibly support the existence of both modes-mirror mode and proton cyclotron wave in the Venus magnetosheath. A number of studies are dedicated to the analysis of power spectra observed in the 


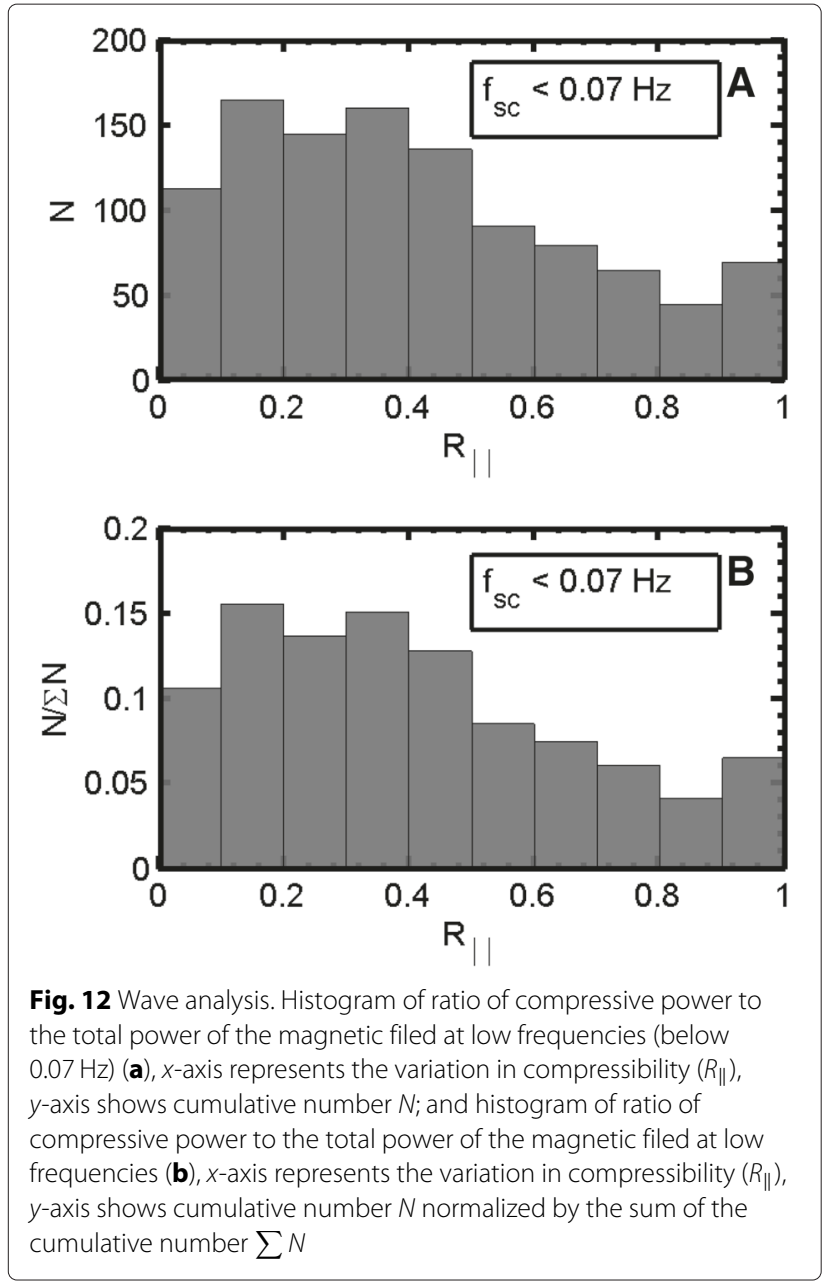

Earth magnetosheath, where not only the mirror mode waves but also Alfvén Ion Cyclotron (AIC) waves, Alfvén vortices (the cylindrical analogue of the nonlinear Alfvén wave) (Alexandrova 2008) are invoked to interpret the spectral break near the proton gyro-frequency.

We consider the break point to be caused by waves in the magnetosheath. One can convert the frequency domain into a wave vector domain using Taylor's frozenin hypothesis. We compute the streamline component of the break point wave vector using the averaged ion flow velocity $\left(V_{\text {flow }}=270 \mathrm{~km} \mathrm{~s}^{-1}\right)$ obtained from ASPERA, ion gyroradius $\left(\rho_{i}=50 \mathrm{~km} \mathrm{rad}^{-1}\right)$ and break point frequency $\left(f_{s c}=0.3 \mathrm{~Hz}\right)$ :

$$
\begin{gathered}
\omega=k V_{\text {flow }} \\
k=\frac{2 \pi f_{s c}}{V_{\text {flow }}} \\
k \rho_{i}=0.3
\end{gathered}
$$

therefore, the observational model shows that the break point appears at a wavenumber $k \rho_{i} \approx 0.3\left(k \rho_{i} \ll 1\right)$. Figure 14 illustrates a schematic representation of the

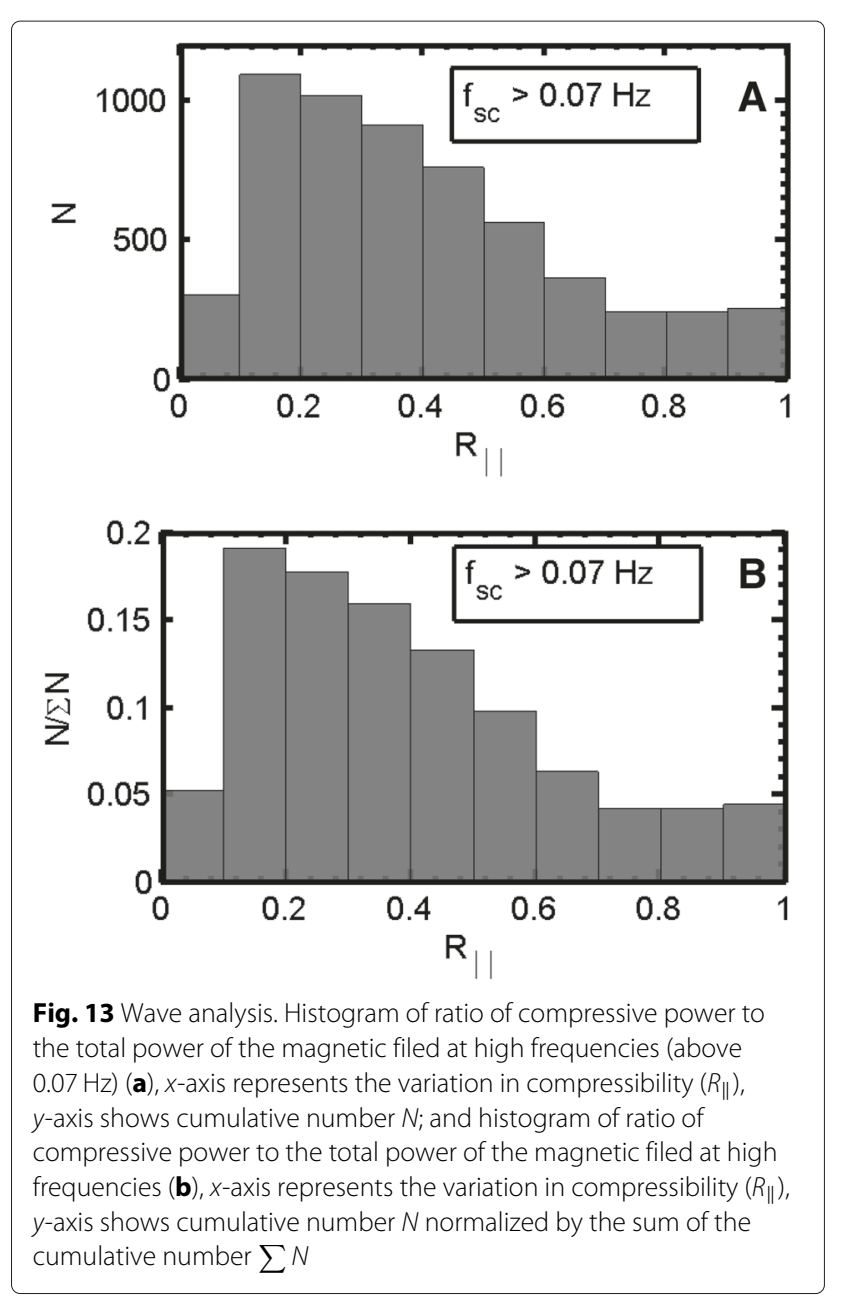

energy spectra of the magnetic field fluctuations, the spectral indices at larger and smaller scales in wavenumber domain, and the break point at $k \rho_{i} \approx 0.3$ accompanied by steepening in the spectra. The energy spectra at larger scale are associated with the mirror mode cascades;

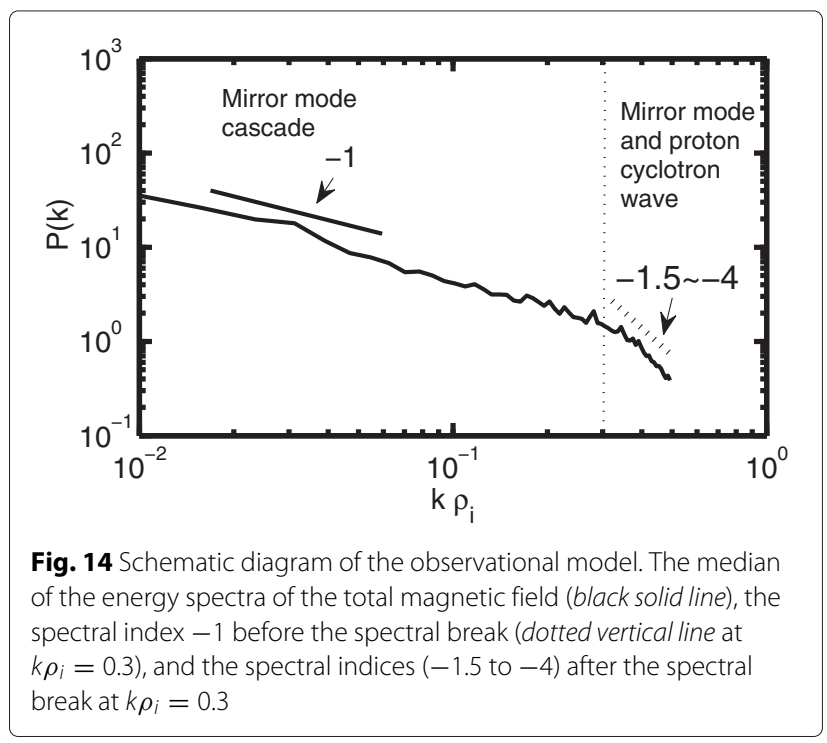


however, the smaller scale energy spectra with significant variation in the spectral indices -4 to -0.5 is a realization of presence of turbulence, mirror mode, and proton cyclotron waves.

The present study is mainly focused on the investigation of the turbulence properties inspirit of search for the power-law behavior in the magnetosheath region. The mirror mode caused by the temperature anisotropy is a likely candidate to explain the low-frequency spectra. We find that at high frequencies, the fluctuations present in the magnetosheath evolve to a turbulent state, in which the spectral index -1.5 can be understood as the slope of the turbulent spectrum in the MHD regime. The spectral index -1.5 found in the present study is also in support to the previous work (Guicking et al. 2010). Guicking et al. (2010) detected the average spectral index of -1.8 of the total power in the magnetosheath. Our investigation is a complementary addition to a previous work (Vörös et al. 2008) in which authors found noisy fluctuations in the magnetosheath as signature of the presence of independent driving sources. Furthermore, the current work is based only on the data sets from 5 months in each year at solar minimum (2006-2008), and a thorough study is required to analyze the data for a complete period of 36 months of solar minimum (2006-2008). In the present investigation, we perform the partial wave analysis in order to give some information about the possible wave modes existence in the magnetosheath. We find both compressive (mirror modes) and non-compressive (proton cyclotron waves) wave interpretations are possible for the magnetosheath waves, but an individual wave mode identification analysis is kept as future research work.

\section{Competing interests}

The authors declare that they have no competing interests.

\section{Authors' contributions}

NKD prepared the manuscript; involved in initiating the research work presented in the manuscript, data selection procedure and data analysis. DS analyzed and interpreted the selected data. YN contributed in physical interpretation of the results as well as structuring the manuscript. PK estimated the power-law spectral index of the energy spectra. ZV contributed in the interpretation. $\mathrm{MD}$ coordinated in the interpretation and manuscript writing. TZ provided the magnetic field data. All authors have analyzed and discussed the results, and approved the final version of the manuscript. Hence, all authors contributed extensively to the work presented in this paper.

\section{Acknowledgements}

The research leading to these results has received funding from the European Community's Seventh Framework Programme under grant agreement $313038 /$ STORM. The work by DS is partially supported by the Austrian Science Fund FWF under grant P25257-N27. We wish to thank M. Volwerk for his valuable suggestions and many helpful contributions.

\footnotetext{
Author details

${ }^{1}$ Space Research Institute, Austrian Academy of Sciences, Schmiedlstrasse 6, Graz, Austria. ${ }^{2}$ University of Graz, NAWI, Graz, Austria. ${ }^{3}$ Geological and Geophysical Institute of Hungary, XIV., Stefánia út 14, 1143 Budapest, Hungary. ${ }^{4}$ Department of Geophysics and Space Science, Eötvös University, Budapest, Hungary.
}

Received: 4 June 2015 Accepted: 12 August 2015

Published online: 25 August 2015

\section{References}

Alexandrova O (2008) Correct wind vs magnetosheath turbulence and Alfvén vortices. Nonlin Processes Geophys 15:95-108

Arthur CW, McPherron RL, Means JD (1976) A comparative study of three techniques for using the spectral matrix in wave analysis. Radio Sci 11:833-845

Barabash S, Sauvaud J-A, Gunell H, Andersson H, Grigoriev A, Brinkfeldt K, Holmstrom M, Lundin R, Yamauchi M, Asamur K, Baumjohann W, Zhang TL, Coates AJ, Linder DR, Kataria DO, Curtis CC, Hsieh KC, Sandel BR, Fedorov A, Mazelle C, Thocaven J-J, Grande M, Hannu M, Koskinen EJ, Kallio E, Sales T, Riihela P, Kozyra J, Krupp N, Woch J, Luhmann J (2007) The analyser of space plasmas and energetic atoms (ASPERA-4) for the Venus Express mission. Planet Space Sci 55:1772-1792

Baumjohann W, Treumann RA, Georgescu E, Haerendel G, Fornaçon K-H, Auster U (1999) Waveform and packet structure of lion roars. Ann Geophys 17:1528-1534

Bavassano Cattaneo MB, Basile C, Moreno G, Richardson JD (1998) Evolution of mirror structures in the magnetosheath of Saturn from the bow shock to the magnetopause. J Geophys Res 103:11961-11972

Biernat HK, Erkaev NV, Arshukova IL, Farrugia CJ, Lammer H, Penz T, Amerstorfer UV, Vogl DF, Zhang TL, Schwingenschuh K, Nakamura R, Baumjohann W, Gunell H, Holmstrom M, Kallio E, Orsini S, Milillo A, Gether K, Leitner M (2005) Aspects of solar wind interaction with Venus. In: Biernat HK, et al. (eds). Proceedings of the Solar-Planetary Relations 2005. Research Signpost, Trivandrum. pp 153-206

Bourouaine S, Alexandrova O, Marsch E (2012) On spectral breaks in the power spectra of magnetic fluctuations in fast solar wind between 0.3 and $0.9 \mathrm{AU}$. Astrophy J 749:102. 7pp

Brace LH, Theis RF, Hoegy WR (1982) Plasma clouds above the ionosphere of Venus and their implications. Planet Space Sci 30:29-37

Constantinescu OD, Glassmeier K-H, Treumann R, Fornaçon K-H (2003) Magnetic mirror structures observed by Cluster in the magnetosheath. Geophys Res Lett 30(15):1802. doi:10.1029/2003GL017313

Delva M, Zhang TL, Volwerk M, Magnes W, Russell CT, Wei HY (2008a) First upstream proton cyclotron wave observations at Venus. Geophys Res Lett 35. L3105

Delva, M, Zhang TL, Volwerk M, Russell CT, Wei HY (2008b) Upstream proton cyclotron waves at Venus. Planet Space Sci 56:1293-1299

Erdös G, Balogh A (1993) Statistical properties of mirror mode structures observed by Ulysses in the magnetosheath of Jupiter. J Geophys Res 101:1-12

Guicking L, Glassmeier K-H, Auster H-U, Delva M, Motschmann U, Narita Y, Zhang TL (2010) Low-frequency magnetic field fluctuations in Venus solar wind interaction region: Venus Express observation. Ann Geophys 28:951-967

Huddleston DE, Strangeway RJ, Blanco-Cano X, Russell CT, Kivelson MG, Khurana KK (1999) mirror mode structures at the Galileo-lo flyby: Instability criterion and dispersion analysis. J Geophys Res 104:17479-17489

Lucek EA, Dunlop MW, Balogh A, Cargill P, Baumjohann W, Georgescu E, Haerendel G, Fornaçon K-H (1999) Mirror mode structures observed in the dawn-side magnetosheath by Equator-S. Geophys Res Lett 26:2159-2162

Luhmann JG, Tatrallyay M, Russell CT, Winterhalter D (1983) Magnetic field fluctuations in the Venus magnetosheath. Geophys Res Lett 10:655-658

Luhmann JG, Russell CT, Elphic RC (1986) Spatial distribution of magnetic field fluctuations in the dayside magnetosheath. J Geophys Res 91:1711-1715

Luhmann JG (1995) The inner magnetosheath of Venus: An analogue for Earth? J Geophys Res 100:12035-12045

McPherron RL, Russel CT, Coleman PJ (1972) Fluctuating magnetic fields in the magnetosphere, 2, ULF waves. Space Sci Rev 13:411-454

Orlowski DS, Russell CT, Krauss-Varban D, Omidi N (1994) A test of the Hall-MHD model: Application to low-frequency upstream waves at Venus. J Geophys Res 99:169-178

Rae I, Mann I, Watt C, Kistler L, Baumjohann W (2007) Equator-S observations of drift mirror mode waves in the dawnside magnetosphere. J Geophys Res A11203. doi:10.1029/2006JA012064

Russell CT, Snare RC, Means JD, Elphic RC, Scarf FL, Brace LH (1980) Pioneer Venus Orbiter fluxgate magnetometer. IEEE T Geosci Remote 18:32-35 
Russell CT, Luhmam J, Elphic RC, Scarf FL, Brace LH (1982) Magnetic field and plasma wave observations in a plasma cloud at Venus. Geophys Res Lett 9:45-48

Scarf F, Neumann S, Brace L, Brace LH, Russell CT, Luhmann JG, Stewart AIF (1985) Current-driven plasma instabilities and auroral-type particle acceleration at Venus. Adv Space Res 5:185-191

Strangeway RJ, Russel CT (1996) Plasma waves and field aligned currents in the Venus plasma mantle. J Geophys Res 101:17,313-17,324

Titov DV, Svedhem H, McCoy D, Lebreton J-P, Barabash S, Bertaux J-L, Drossart P, Formisano V, Haeusler B, Korablev OI, Markiewicz W, Neveance D, Petzold M, Piccioni G, Zhang TL, Taylor FW, Lellouch E, Koschny D, Witasse O, Warhaut M, Acomazzo A, Rodrigues-Cannabal J, Fabrega J, Schirmann T, Clochet A, Coradini M (2006) Venus Express: Scientific goals, instrumentation, and scenario of the mission. Cosmic Res 44:334

Tsurutani BT, Smith EJ, Anderson RR, Ogilvie KW, Scudder JD, Baker DN, Bame SJ (1982) Lion roars and nonoscillatory drift mode mirror waves in the magnetosheath. J Geophys Res 87:6060-6072

Volwerk M, Zhang TL, Delva M, Voros Z, Baumjohann W, Glassmeier K-H (2008a) Mirror-mode-like structures in Venus induced magnetosphere. J Geophys Res 113:E00B16

Volwerk, M, Zhang TL, Delva M, Voros Z, Baumjohann W, Glassmeier K-H (2008b) First identification of mirror mode waves in Venus magnetosheath? Geophys Res Lett 35:L12204

Vörös Z, Zhang TL, Leubner MP, Volwerk M, Delva M, Baumjohann W (2008) Intermittent turbulence, noisy fluctuations, and wavy structures in the Venusian magnetosheath and wake. J Geophys Res 113:E00B21

Welch PD (1967) The use of fast Fourier transform for the estimation of power spectra: A method based on time averaging over short, modified periodograms. IEEE Trans Audio Electroacost AU 15:7073

Zhang TL, Baumjohann W, Delva M, Auster H-U, Balogh A, Russell CT, Barabash S, Balikhin M, Berghofer G, Biernat HK, Lammer H, Lichtenegger H, Magnes W, Nakamura R, Penz T, Schwingenschuh K, Voros Z, Zambelli W, Fornacon K-H, Glassmeier K-H, Richter I, Carr C, Kudela K, Shi JK, Zhao H, Motschmann U, Lebreton J-P (2006) Magnetic field investigation of the Venus plasma environment: Expected new results from Venus Express. Planet Space Sci 54:1336-1343

Zhang TL, Delva M, Baumjohann W, Volwerk M, Russell CT, Barabash S, Balikhin M, Pope S, Glassmeier K-H, Wang C, Kudela K (2008) Initial Venus Express magnetic field observations of the magnetic barrier at solar minimum. Planet Space Sci 56:790-795

\section{Submit your manuscript to a SpringerOpen ${ }^{\odot}$ journal and benefit from:}

- Convenient online submission

- Rigorous peer review

- Immediate publication on acceptance

- Open access: articles freely available online

- High visibility within the field

- Retaining the copyright to your article

Submit your next manuscript at $\boldsymbol{\triangleright}$ springeropen.com 